Meta

Journal des traducteurs

Translators' Journal

\title{
Le chaos de la traduction et la traduction du chaos
}

\section{Pier-Pascale Boulanger}

Volume 51, numéro 1, mars 2006

URI : https://id.erudit.org/iderudit/012997ar

DOI : https://doi.org/10.7202/012997ar

Aller au sommaire du numéro

Éditeur(s)

Les Presses de l'Université de Montréal

ISSN

0026-0452 (imprimé)

1492-1421 (numérique)

Découvrir la revue

Citer cet article

Boulanger, P.-P. (2006). Le chaos de la traduction et la traduction du chaos. Meta, 51(1), 119-130. https://doi.org/10.7202/012997ar

\section{Résumé de l'article}

Les affinités sont étonnantes entre les constatations épistémologiques que la théorie du chaos a amené les sciences dites exactes à faire et une pensée de la traduction libérée d'une conception fixiste du sens. Penser la traduction en passant par la théorie du chaos, c'est surtout annuler les dichotomies inopérantes échues des conceptualisations traditionnelles, visée participant des discours de la " troisième voie ». Non-linéarité, sensibilité critique aux conditions initiales et géométrie fractale sont autant de thèmes qui potentialisent la théorisation de la traduction dans une épistémologie tablant sur la mouvance et la subjectivité des paramètres d'interprétation, sur l'instabilité persistante entre l'ordre et le désordre, sur la valeur heuristique donc positive du désordre et sur l’incomplétude.
Ce document est protégé par la loi sur le droit d'auteur. L'utilisation des services d'Érudit (y compris la reproduction) est assujettie à sa politique d'utilisation que vous pouvez consulter en ligne.

https://apropos.erudit.org/fr/usagers/politique-dutilisation/ 


\title{
Le chaos de la traduction et la traduction du chaos
}

\author{
PIER-PASCALE BOULANGER \\ Université Concordia, Montréal, Canada \\ pierpa@sympatico.ca
}

\begin{abstract}
RÉSUMÉ
Les affinités sont étonnantes entre les constatations épistémologiques que la théorie du chaos a amené les sciences dites exactes à faire et une pensée de la traduction libérée d'une conception fixiste du sens. Penser la traduction en passant par la théorie du chaos, c'est surtout annuler les dichotomies inopérantes échues des conceptualisations traditionnelles, visée participant des discours de la «troisième voie». Non-linéarité, sensibilité critique aux conditions initiales et géométrie fractale sont autant de thèmes qui potentialisent la théorisation de la traduction dans une épistémologie tablant sur la mouvance et la subjectivité des paramètres d'interprétation, sur l'instabilité persistante entre l'ordre et le désordre, sur la valeur heuristique donc positive du désordre et sur l'incomplétude.
\end{abstract}

\begin{abstract}
The affinities that exist between the epistemological observations of the so-called "exact" sciences arrived at via the chaos theory, on the one hand, and the notion of translation as being free of a fixist conception of meaning, on the other hand, are remarkable indeed. To consider translation in light of the chaos theory is above all to do away with the ineffective dichotomies stemming from traditional conceptualizations, an aim partaking of the discourse of the "third path." Nonlinearity, critical sensitivity to initial conditions, fractal geometry - these are just so many themes potentializing the theorization of translation into an epistemology based on the ever-changing, subjective nature of the parameters of interpretation, on the state of persistent instability between order and disorder, on the heuristic and therefore positive value of disorder, and on incompleteness.
\end{abstract}

\section{MOTS-CLÉS/ KEYWORDS}

épistémologie de l'ouverture, théorie du chaos, système dynamique, pensée du désordre

Tohu-bohu; confusion; bouleversement. Voilà autant de renvois sémantiques qui se greffent à l'idée de chaos. Du tohou vabohou hébreu, tohu-bohu désigne l'état informe et vide de la terre avant la création ${ }^{1}$. Cet état premier, voire primitif, de l'univers est à comprendre comme la confusion d'un monde n'ayant pas encore reçu la vie de Dieu. Dans son deuxième sens, le chaos est synonyme de désordre grave, à comprendre cette fois-ci, au contraire d'un vide, comme un trop-plein de vie ou d'énergie. Cet état chaotique des choses reçoit bien souvent une valeur négative. Devant l'ambivalence de ce mot, signifiant à la fois vide et trop-plein, il faut bien admettre la performativité de celui-ci: le mot chaos est chaotique. Et le chaos qui nous intéresse est davantage le phénomène de désordre qui a capté l'attention de physiciens et de mathématiciens dès la fin des années 1960. Parce qu'ils dépassent les principes de physique newtonienne, fondée sur la dynamique à deux corps, les phénomènes chaotiques ont longtemps été mis de côté par la physique classique, en tant qu'ils défiaient la loi normale de l'erreur ${ }^{2}$. En fait, la théorie du chaos était à la physique ce que la traduction était 
à la linguistique, c'est-à-dire aberrante. La théorie du chaos et la théorie de la traduction ont donc ceci en commun qu'elles ont été mises à l'écart, car elles compromettaient l'ordre établi des principes fondateurs (mécaniques et linguistiques), portant en elles ce potentiel dynamique et subversif d'un système non linéaire. Par ailleurs, la théorie du chaos et la traductologie invitent toutes deux à une recherche interdisciplinaire, dont les nouvelles alliances dépassent la science normale, qui, selon Thomas Kuhn (1983), s'attache davantage à énoncer et à résoudre des problèmes dans l'espace clos de sa tradition théorique. Les affinités sont étonnantes entre les constatations épistémologiques que la théorie du chaos a permis aux sciences dites exactes de faire et une pensée de la traduction libérée d'une conception fixiste du sens. Dans le sillage des travaux post-structuralistes et déconstructionnistes, les théories de la traduction sont de plus en plus nombreuses à dire tout haut la mouvance du sens, le métissage des langues, le brouillage des identités nationales, cette turbulence socioculturelle inouïe que l'activité traductive donne à voir. Le temps est venu de reconnaître le pouvoir heuristique du désordre et, conséquemment, la valeur positive qui peut lui être accordée.

Une mise en garde s'impose, toutefois, à savoir qu'il n'est pas question ici de translater aveuglément l'appareil théorique qui entoure le chaos vers le domaine de la traduction. Plutôt, en prenant appui sur l'interdisciplinarité telle que Berman la conçoit, il s'agit de penser la traduction en passant par le chaos. Et ce, dans le but de continuer à pluraliser le savoir traductif et à renforcer l'idée selon laquelle «la traductologie n'est pas un discours fermé qui prendrait en vue tel champ du réel: justement, l'aire de la traduction n'est pas un "champ", au sens que prend ce concept dans les sciences» (Berman 1989: 676). La traductologie gagne toujours à tisser des liens novateurs avec des modes de pensée autres. Aussi, montrer la pertinence de la théorie du chaos dans une réflexion sur la traduction, c'est prouver la force de cette dernière de toujours repousser ses limites théoriques, c'est-à-dire contrer le statisme qui guette tout système conceptuel.

\section{LA THÉORIE DU CHAOS}

En 1900, Max Planck pose sa théorie des quanta d'énergie, lesquels forment les particules sous-atomiques (les quarks, dont les électrons) ainsi que les liens entre elles (les gluons, dont les photons). Planck découvre alors que le monde du très petit n'obéit pas aux règles de la physique classique déterminant le comportement des choses perceptibles ${ }^{3}$. La révolution scientifique produite par la théorie des quanta engage la physique, non sans résistance, vers la désuniversalisation de ses principes fondamentaux: les lois de Newton ne permettent pas de prédire le comportement des particules. C'est dans cette ouverture épistémologique que l'étude des phénomènes chaotiques vient s'inscrire quelque 70 ans après Planck.

La théorie du chaos est le grand titre sous lequel diverses sciences étudient les systèmes non linéaires, apériodiques ou complexes, dits dynamiques et ouverts, en tant qu'ils peuvent se transformer à l'infini sans jamais retrouver leur état initial. Il en va ainsi du système météorologique, de la configuration des flocons de neige ${ }^{4}$, du comportement des fluides en ébullition et du parcours migratoire des oiseaux, par exemple. On peut aisément ajouter à cette liste la dynamique transformatoire du sens qui caractérise l'activité traductive, telle que Derrida la met en lumière dans Positions, où il dit: 
[...] à la notion de traduction, il faudra substituer une notion de transformation: transformation réglée d'une langue par une autre, d'un texte par un autre. Nous n'aurons et n'avons en fait jamais eu affaire à quelque «transport» de signifiés purs que l'instrument - ou le «véhicule» - signifiant laisserait vierge et inentamé, d'une langue à l'autre, ou à l'intérieur d'une seule et même langue (1972: 31).

Derrida soutient que le sens est fondé sur le jeu interminable de différences entre les marques de la langue et n'est jamais présent comme tel ni donné d'avance, mais toujours advenant et différé; c'est ce qu'il nomme la différance ${ }^{5}$. Quand on les entend, les expressions «terre inconnue» et «terrain connu» ne sont-elles pas pareilles et contraires à la fois? Voilà qui montre bien simplement le désordre potentiel du langage. Et si le sens est mouvant, tel que la différance permet de le comprendre, la traduction est ce qui montre le mieux le déséquilibre de celui-ci, qui advient du jeu des marques linguistiques entre elles. L'instabilité du sens se trouve décuplée, voire potentialisée, lorsque des textes, des langues et des sensibilités culturelles et historiques entrent en interaction dans le travail de traduction. Aussi, de nombreux discours contemporains reconnaissent à la traduction un potentiel dynamique, ce qui vient mettre fin à la prédominance de la conception traditionnelle selon laquelle la traduction doit établir un rapport d'adéquation linéaire entre des contenus figés de langue qui constitueraient des textes, idéalisés comme des systèmes fermés.

Sur le plan mathématique, les systèmes dynamiques ont ceci de particulier qu'ils excluent toute possibilité d'une seule bonne réponse finale, d'où le phénomène chaotique. Le rapprochement avec l'idée qu'un seul texte peut générer de multiples versions adéquates est derechef pertinent pour la traduction. Loin d'être un champ uniforme, la recherche scientifique du chaos donne cours à deux paradigmes qui coexistent et poursuivent certains axiomes communs, tout en utilisant des méthodes mathématiques différentes. Dans le premier paradigme, le chaos est conceptualisé comme un état qui précède l'ordre. Dans cette optique, les scientifiques s'engagent à chercher les structures ordonnées qui émergent du chaos. Les conclusions sont aisément extrapolées dans le domaine philosophique. Ce paradigme suit largement la pensée d'Ilya Prigogine, qui a élaboré la théorie des structures dissipatives, reprise par Mitchell Feigenbaum, selon laquelle les turbulences sont modélisables, puisqu'elles suivent un parcours prévisible jusqu'à l'état de chaos. Reconnue par un prix Nobel en 1977, la théorie de Prigogine postule que la turbulence des systèmes instables crée de l'entropie, c'est-à-dire une perte d'énergie causée par le mouvement aléatoire des particules, ce qui permet l'évolution des systèmes vers un état d'équilibre. Dans le deuxième paradigme, les chercheurs croient qu'il y a un ordre caché dans les systèmes chaotiques, ce que révèlent les attracteurs étranges ${ }^{7}$. Ces représentations graphiques montrent qu'en se dissipant à l'infini les systèmes chaotiques tendent néanmoins à s'organiser en des points qui se concentrent dans une région particulière sans toutefois se retrouver deux fois à la même place. Malgré la dynamique dissipative, les points semblent s'unir et générer des figures complexes dans l'espace. Des chercheurs tels Edward Lorenz et Benoît Mandelbrot sont au nombre des premiers qui se sont intéressés aux systèmes dynamiques demeurant chaotiques et à leur pouvoir de générer de l'information toujours nouvelle. C'est dans ce paradigme que la traduction semble pouvoir être pensée. Les caractéristiques des systèmes chaotiques qui intéressent la traduction sont théorisables sous trois principaux thèmes: la non-linéarité, la sensibilité critique aux conditions initiales et les formes fractales. 


\section{Non-linéarité}

Sur le plan mathématique, un système non linéaire est dit ouvert en ce qu'il rend impossible le retour aux donnes posées en début de calcul. Parce qu'un système linéaire permet la réciproque $(1+2=3-2=1)$, il est dit fermé. Pour illustrer ce qu'est un système ouvert, on peut s'imaginer deux moitiés équivalentes de sable juxtaposées dans une boîte, soit une moitié blanche et l'autre noire. Les deux moitiés sont composées de la même matière, du sable. Si on brasse le contenu de la boîte 20 coups dans le sens des aiguilles d'une montre, on se rend vite compte qu'il devient difficile de trancher entre la zone blanche et la zone noire, puisque la masse de sable s'est mélangée et est devenue grise. Si on brasse ensuite 20 coups dans le sens inverse avec le même bâton et la même intensité, on n'arrive pas du tout à reconstituer les zones blanche et noire initiales et on obtient plutôt un mélange encore plus important de la zone grise. Il en va ainsi d'un système ouvert, dont la non-linéarité induit une dynamique chaotique qui empêche le retour aux données initiales. Si on extrapole cette logique au mouvement traductif, on peut poser que les opérations qui ont servi à traduire le texte $\mathrm{A}$ vers le texte $\mathrm{B}$ ne seront pas les mêmes qui seront utilisées pour retraduire le $B$ vers le texte $A$. On comprend vite qu'il est impossible de restituer le texte $A$ original en traduisant le texte $B$, parce qu'un texte est un système ouvert dans lequel les variables sont nombreuses et subissent l'influence de phénomènes non maîtrisables (la subjectivité de l'interprétant, les limites de la langue, les conditions de travail...). Les résultats demeurant imprédictibles, on dit du système qu'il est chaotique. Envers et contre toutes les prédictions de la physique classique, la théorie du chaos montre que la nature n'agit pas de manière linéaire, mais plutôt selon des configurations qui résultent d'un ensemble d'impulsions imperceptibles: le propre du chaos est l'émergence et le renouvellement. Ne sont-ce pas là les principaux catalyseurs de la traduction en ce qui a trait aux sens d'un texte?

\section{Sensibilité critique aux conditions initiales}

Dans les équations linéaires qui expliquent le fonctionnement des systèmes équilibrés, la loi normale de l'erreur prévoit que d'infimes fluctuations subies en début de calcul influent de manière infime sur les résultats. Cela se confirmerait également à plus grande échelle. En revanche, dans le comportement non linéaire des systèmes turbulents, une cause très petite passant inaperçue entraîne des effets considérables sur les résultats, du fait que l'interaction interminable de nombreux facteurs amplifie une fluctuation survenue dans l'évolution du système ${ }^{8}$. Sur le plan théorique, cette disproportion entre la cause et l'effet vient ébranler le statut universel de la loi normale de l'erreur, devenue applicable spécifiquement aux systèmes linéaires de la physique classique ${ }^{9}$. Ainsi, la non-linéarité du lien de causalité que les systèmes chaotiques révèlent s'explique par la sensibilité critique aux conditions initiales ${ }^{10}$. Selon cette idée, à moins que les conditions initiales ne soient précisées à l'infini, les systèmes apériodiques, non linéaires ou complexes deviennent vite imprévisibles. Il n'y a qu'à penser à la météo et à l'économie, tout à fait imprévisibles au-delà de deux ou trois jours, malgré la compilation de quantités effarantes d'antécédents. Les systèmes chaotiques confirment que les influences imperceptibles ne peuvent pas être négligées. Il s'agit là du principe d'incertitude, s'il en est un, qui empêche l'exactitude: les conditions 
initiales d'un système complexe ne pouvant être déterminées, l'évolution de celui-ci ne peut pas être prédite exactement. Ce principe trouve une pertinence sans contredit dans le domaine de la traduction, où la condition initiale que serait le sens du texte original n'est toujours qu'approximative, en tant qu'il porte en lui le potentiel d'interprétations multiples. Dans cette perspective, il est donc juste d'affirmer que le processus traductif est chaotique, du fait qu'il ne peut jamais connaître avec une précision infinie les conditions initiales. Derrida et de nombreux autres théoriciens de la dynamique transformatoire de la langue ont déjà souligné ce phénomène.

Parce qu'ils sont à la fois déterministes et imprévisibles, les systèmes chaotiques sont dits paradoxaux: d'une part, ils exigent des paramètres infiniment précis, ce qu'aucune intelligence humaine ni artificielle ne peut garantir; d'autre part, leurs résultats demeurent imprédictibles, parce que changeants. Ce paradoxe donne lieu à une troisième voie en dehors du schéma dualiste traditionnel selon lequel l'ordre et le désordre doivent mutuellement s'exclure. En outre, les études sur la turbulence des fluides ${ }^{11}$ ont révélé que les zones de mouvement aléatoire coexistent toujours avec des zones de mouvement régulier: dans un tourbillon, il y a toujours des îlots de stabilité, mais qui changent sans cesse. De même, dans un texte, il se trouve des zones de calme, soit un signifiant quasi-inchangeant, tels un chiffre ou une date. Peu importe le nombre de fois que le texte serait traduit, ces données resteraient les mêmes. Quant aux zones de turbulence, elles surviennent dans un texte là où les degrés d'interprétation augmentent. Si les travaux déconstructionnistes ont permis d'affirmer que la nature du sens est plurielle, la théorie du chaos permet aujourd'hui de dire qu'elle est chaotique, mais aussi que l'ordre coexiste avec le désordre.

\section{Formes fractales}

Représentations numériques, les formes fractales cartographient les résultats de computations électroniques d'algorithmes qui peuvent se réitérer à l'infini (la récursivité) et générer des images fascinantes. Mandelbrot ${ }^{12}$, s'intéressant à la conceptualisation géométrique de problèmes mathématiques, s'est servi de l'ordinateur pour générer une géométrie fractale ${ }^{13}$, qui ouvre sur des ramifications quasi-infinies. En fait, une forme fractale à proprement parler présente une longueur infinie dans un espace fini ${ }^{14}$. Sur le plan mathématique, une séquence initiale finie (10 caractères suffisent à générer l'ensemble de Mandelbrot) peut générer des figures infinies. Émile Benveniste n'a-t-il pas dit la même chose du langage, à savoir qu'un matériau fini génère des possibilités infinies? À partir de ces idées, n'apparaît-il pas qu'un texte en traduction est une forme fractale? La traduction ne montre-t-elle pas des développements sémantiques infinis à l'intérieur d'un espace textuel fini?

De surcroît, la géométrie fractale «permet de mesurer des qualités qui autrement n'auraient pas de définition claire: le degré de rugosité, de fragmentation, d'irrégularité d'un objet» (Gleick 1991: 131). À cet égard, les formes fractales défient les plans lisses et linéaires de la géométrie euclidienne ${ }^{15}$.

La nouvelle géométrie donne de l'univers une image anguleuse et non arrondie, rugueuse et non lisse. C'est une géométrie du grêlé, du criblé, du disloqué, du tordu, de l'enchevêtré, de l'entrelacé (Gleick 1991: 127).

En fait, Mandelbrot se rend compte que la mesure d'un objet est relative ${ }^{16}$, donc qu' elle dépend du point de vue et de l'échelle à partir desquels elle a été obtenue. Une forme 
fractale représente visuellement ce qui arrive lorsque les dimensions sont fractionnées, tel que la construction du flocon de Koch ${ }^{17}$ ou du cube de Sierpinski-Menger ${ }^{18}$ permet de le constater: en de nombreux points d'une forme fractale, il est possible de retrouver une configuration qui rappelle la forme globale. En ce sens, les formes fractales sont symétriques à différentes échelles. La géométrie du chaos pluralise les dimensions et les échelles à un point tel qu'il n'est plus possible de croire en l'universalité d'une mesure. Plus l'outil de mesure est petit, plus la mesure grossit; cela ruine l'univocité de toute réponse et amène la science à admettre sa part de subjectivité.

À bien comprendre la nature du chaos, la traduction semble appartenir à la catégorie des systèmes dynamiques chaotiques ou, du moins, elle gagne à être pensée dans l'ouverture épistémologique de la théorie du chaos. Il ne faut pas oublier que malgré les connaissances poussées que les nombreuses branches de la linguistique ont pu acquérir, le comportement linguistique demeure imprévisible. Et malgré la finitude du matériau graphique de base que sont les mots et les phrases du texte original, le mouvement interprétatif de la traduction n'est jamais connu d'avance ni régulé. On entrevoit ici le chaos de la traduction. Il semble, effectivement, que la traduction réponde à la définition du chaos, c'est-à-dire cet état d'instabilité persistante entre l'ordre et le désordre. En somme, la traduction ne peut plus être conceptualisée en termes de rapport linéaire entre les mots d'une langue A et ceux d'une langue B. Aussi est-il valable d'ouvrir de nouvelles pistes réflexives en transposant à la traductologie quelques propositions émanant de la théorie du chaos.

\section{Le chaos de la traduction}

«[...] le chaos est une science des processus plutôt que des états, une science du devenir plutôt que de l'étant» (Gleick 1991: 20). Les transformations et les récursivités occupent principalement l'étude des systèmes dynamiques. Certains se penchent sur la transformation des récursivités, où les choses semblent se répéter, mais jamais exactement de la même manière, du fait qu'il survient une modulation qui transforme le comportement du système. Il en va ainsi des attracteurs étranges. Tandis que d'autres étudient la récursivité des transformations, selon laquelle les formes semblent se complexifier à l'infini, mais toujours à partir d'une même donne de base. On peut penser à la ramification des formes fractales. La pertinence de la première perspective pour la traduction semble aller d'elle-même: le texte traduit succède au texte original, mais ne lui ressemble pas. Quant à la deuxième perspective, on peut concevoir tout texte selon l'optique multidimensionnelle propre à la géométrie du chaos: à différentes échelles, un texte prend des formes particulières et ouvre sur des perspectives différentes. La traductologie devient le terrain fertile d'une telle conceptualisation et doit pouvoir tolérer la coexistence de nombreux points de vue, sans qu'il soit nécessaire de légiférer sur une mesure universelle que serait le sens d'un texte ou une théorie générale de la traduction.

«Le chaos pose des problèmes défiant la méthodologie scientifique classique» (Gleick 1991 : 132-133). On dit qu’il a renouvelé la science. La physique traditionnelle, celle qui enseigne les principes de Newton et qui traite les systèmes non linéaires comme autant d'aberrations théoriques à ces lois de la nature, a confondu connaître la composition d'un objet et prédire le comportement de celui-ci. Convaincue de la finitude de son savoir, la physique classique a déduit à tort qu'en connaissant tous les 
éléments d'un système et en les soumettant aux lois fondamentales, elle pouvait prévoir l'évolution de ce dernier. C'est là également l'erreur déterministe de la linguistique structurale (que Benveniste a cernée) qui a cherché à prédire le comportement du système linguistique à partir des connaissances profondes qu'elle avait de ses composantes morphologiques, lexicales et syntaxiques. Les théories traditionnelles de la traduction sont fortes de ce fondement épistémologique de la science, selon lequel comprendre a le sens de connaître à l'avance les résultats. Pour simplifier à l'extrême, cela impliquerait que la connaissance des éléments du texte original et l'application de règles de traduction (prenons celles de Vinay et Darbelnet) à ceux-ci, devraient permettre d'arriver à un nombre de traductions toujours plus restreint d'un texte, moyennant quelques variations attribuables à la loi normale de l'erreur. C'était là en effet le mandat des premières théories de la traduction. Mais en tant que système dynamique ou non linéaire, la traduction empêche une telle illusion scientiste. Dès lors que la dimension chaotique du comportement traductif est reconnue et théorisée de manière positive, il n'y a plus lieu de chercher à conformer les processus de traduction à des principes universels en vue de converger vers une solution unique. Ce sont là des critères de scientificité que les théories traditionnelles (d'orientation linguistique) ont calqués des sciences exactes. Chaque situation de traduction appelle un savoir qui lui est spécifique, non connu d'avance.

Plus un système est chaotique, plus il est riche en information - plus il en produit $^{19}$. La science des systèmes dynamiques accorde au chaos une valeur positive. Il n'est plus conçu en termes d'absence et de vide, mais plutôt comme une source inépuisable d'information qui repousse les limites épistémologiques de la science. La traduction est une activité qui potentialise les différents degrés de sens, ce que chaque retraduction confirme. Et si les textes littéraires sont davantage retraduits que les textes pragmatiques, c'est qu'ils se comportent comme les formes fractales, qui se ramifient un plus grand nombre de fois. En tant que système chaotique, la traduction met au défi l'entropie dont tout le discours culpabilisant de la perte l'accuse. À la limite, l'entropie a ceci de positif, qu'elle permet à un système turbulent de retrouver un état d'équilibre, qui correspondrait sur le plan de la traduction à la publication du texte traduit (dont il a bien fallu arrêter le sens).

«On découvre que les systèmes les plus simples posent des problèmes de prédictibilité extraordinairement difficiles. Pourtant, dans ces systèmes, l'ordre surgit spontanément - l'ordre et le désordre» (Gleick 1991: 23). On découvre la complexité du quotidien, se rendant compte du paradoxe de la science qui peut prédire le comportement des phénomènes d'ordre infiniment grand (telle la masse d'un objet aux confins de notre galaxie) ${ }^{20}$ et infinitésimalement petit (tel le comportement de particules sous-atomiques), mais non la trajectoire d'un nuage de crème versée dans une tasse de café brûlant, ni le climat terrestre, ni le cours de l'économie. La théorie du chaos montre de manière percutante qu'il n'y a rien d'ordinaire au quotidien. Sur le plan épistémologique, cette constatation met fin au présupposé fondateur de la physique newtonienne, selon lequel la linéarité et l'ordre constituent la nature des choses. Et on se rend compte que le chaos n'est plus à penser selon un rapport de négation de l'ordre, tel que l'illustre la syntaxe du terme «non-linéarité» (c'est pourquoi l'expression «système ouvert» est plus juste). La science se voit donc appelée à problématiser le désordre à l'extérieur du schéma binaire et réducteur, puisque la spécificité théorique du chaos transgresse les lois traditionnellement déterministes. Cette transformation 
épistémologique n'est pas sans rappeler la pensée de l'altérité, héritière de la philosophie de la décolonisation et de la déconstruction, où l'Autre est autre que le non-moi, grand catalyseur de la pensée postmoderne. En fait, la traduction peut être pensée comme un espace dynamique où des langues se rencontrent et interagissent, en harmonie ou en interférence, pour former une langue en traduction ${ }^{21}$, qui n'est ni tout l'autre ni tout le même. Cet espace se donne à penser comme une source de possibilités de langue toujours renouvelées.

\section{ATTENTION AU CALQUE ÉPISTÉMOLOGIQUE}

Pour autant qu'il soit tentant de translater la théorie du chaos vers la traductologie et de l'appliquer en bloc à la théorisation de la traduction, une telle manœuvre présente certains risques de calque épistémologique. En outre, la théorie du chaos n'est pas l'Eldorado théorique vers lequel il faudrait s'exiler: elle n'annule pas les perspectives totalisantes, puisque la physique s'en sert pour modéliser le désordre (la turbulence), entreprise félicitée par le comité du prix Nobel en 1977, en vue de le maîtriser et de l'enrayer. Ce type de recherche se voit généreusement financé, lorsque par exemple ses applications servent directement à maximiser l'écoulement d'un fluide dans une pipeline ou à réduire la turbulence aérodynamique sur les ailes d'un avion. Il ne faut donc pas s'y méprendre: la théorie du chaos ne ruine pas d'emblée l'entreprise rationnelle et totalisante de la science. Les études de la boîte noire en traductologie s'inscrivent dans ce sillage théorique, dans la mesure où elles admettent la dimension chaotique de l'activité traductive, mais espèrent en modéliser le processus, afin d'en maîtriser l'output. Sur le plan épistémologique, par ailleurs, la théorie du chaos continue de s'appuyer sur la mécanique newtonienne et la géométrie euclidienne, ne serait-ce que partiellement et pour les dépasser. Il devient illusoire d'affirmer une rupture théorique nette, bien qu'une révolution dans la manière antinomique de concevoir l'ordre et le désordre se soit bel et bien produite. Il y a toujours un jeu entre les nouvelles idées et les formations théoriques plus anciennes.

Outre cette mise en garde, il est permis de constater le dialogue qui existe entre la théorie du chaos et les sciences humaines ${ }^{22}$. Pour autant que les résultats générés par les recherches sur les systèmes ouverts révèlent de nouvelles pistes conceptuelles aux sciences humaines, il ne faut pas croire que celles-ci réfléchissent à la remorque des sciences exactes. Le chaos est un concept à forte charge culturelle et littéraire: certains auteurs en trouvent la source dans les textes d'Hésiode ${ }^{23}$. Il n'est pas fortuit non plus de rappeler que le relativisme radical trouve ses précurseurs en Nietzsche, entre autres penseurs, bien avant la parution de l'article phare de Lorenz, «Deterministic Nonperiodic Flow», en 1963. L'idée de dialogue exprime donc plus justement l'interdépendance des rapports entre la science et le quotidien qui occupe également aujourd'hui ${ }^{24}$ les sciences humaines. La science du chaos présente des affinités épistémologiques avec la traductologie, car elle admet l'intuition comme vecteur de scientificité, forte du pressentiment que le désordre recèle une valeur heuristique qui excède la mise en boîte traditionnelle des phénomènes chaotiques. En outre, elle accorde la légitimité théorique à l'esthétique des figures géométriques du chaos, ruinant ainsi l'opposition dualiste classique entre les sciences exactes et les sciences humaines. Le brouillage des frontières autrefois bien définies entre ces deux sphères d'activité déterritorialise les zones conceptuelles et théoriques traditionnellement cloisonnées. 


\section{LA TRADUCTION DU CHAOS}

Comment le chaos se traduit-il en traductologie? Quelles en sont les implications épistémologiques? Tout comme la théorie du chaos s'est traduite en biologie par une conceptualisation fractale des organes humains et en économie par la reconnaissance de l'apériodicité des fluctuations des marchés, le chaos vient renforcer le pressentiment de la traduction quant à la valeur heuristique de son activité dynamique et ouverte. Ainsi, la traductologie se décharge de la requête scientiste d'un rapport linéaire (ou de l'équivalence parfaite) entre texte original et texte traduit. Depuis que la géométrie des formes fractales a mis en lumière la relativité des mesures et la multiplicité des échelles, le relativisme théorique confirme la nature locale, subjective et mouvante des paramètres d'analyse quels qu'ils soient. Par ailleurs, la rugosité, la fragmentation et la complexité des formes qui intéressent la géométrie fractale rappellent l'éclatement des langues et l'ouverture du sens. Transposée à la traduction, cette conceptualisation donne à voir les multiples dimensions d'un texte, faisant en sorte que plus on s'en approche, plus on en découvre les contours irréguliers, tel l'escargot de Lorenz, qui arpente la côte britannique. Malgré tous les degrés d'interprétation possibles d'un texte, on constate à la lecture de celui-ci qu'il se dessine une symétrie d'échelle ou un ordre, qui correspond au principe organisateur qui fait fonctionner un texte comme système tel qu'Henri Meschonnic l'entend ${ }^{25}$. La théorie du chaos devient d'autant plus pertinente pour une pensée de la traduction qu'elle propose une troisième voie, selon laquelle le schéma binaire traditionnel opposant l'ordre au désordre devient inopérant ${ }^{26}$. On peut même conceptualiser le texte traduit comme une forme fractale, qui se caractérise par la pluralité et la complexité de ses échelles sémantiques et les diverses configurations qui peuvent en ressortir. Sur le plan de la recherche scientifique, le chaos est novateur et constructif, car il produit des résultats inattendus et souvent inédits, relançant ainsi la recherche. Cela rejoint l'idée que la traduction est ce qui dans la langue permet de renouveler les formes d'écriture et pluraliser les perspectives. Par conséquent, le chaos se voit accorder une valeur positive, dans la mesure où sa dynamique non linéaire et ses systèmes turbulents indiquent de nouvelles pistes de recherche. Cette affirmation semble aisément transposable au processus traductif.

La théorie du chaos a amené la science à admettre que comprendre ne peut plus avoir exclusivement le sens de prédire. C'est là un des principaux effets de levier qui a permis à la traduction de critiquer l'entreprise scientiste de la linguistique et l'a motivée à se libérer de son emprise théorique. Par ailleurs, la théorie du chaos montre qu'il n'y a rien de simple au quotidien en explorant la thermodynamique, l'économie et la météorologie, ainsi que les configurations fractales, comme les plantes, les organes humains et les reliefs terrestres. Cet intérêt que manifeste la théorie du chaos à l'égard des choses qui parsèment le quotidien humain rappelle les projets de traduction féministes et postcolonialistes, fondés sur l'hypersubjectivisation du sens, par laquelle le sujet traduisant joue pleinement sur la dimension locale de sa situation linguistique, sociale et culturelle.

Enfin, le chaos supprime les frontières entre les disciplines scientifiques. Il réunit mathématiciens, biologistes, chimistes, physiciens, économistes, statisticiens, etc. L'interdisciplinarité qu'appelle la théorisation du chaos fait interagir des disciplines traditionnellement cloisonnées ${ }^{27}$ selon une dynamique théorique transversale qui 
caractérise également certaines théories de la traduction. Pour preuve, l'interaction des Cultural Studies, de la sociologie, de la déconstruction, des études féministes et postcolonialistes, de la philosophie, de la psychanalyse, de l'herméneutique, de la poétique et, en l'occurrence, de la théorie du chaos avec la traductologie a donné lieu à des discours novateurs sur la traduction. Cet enrichissement théorique présuppose un espace conceptuel ouvert, voire infini. Et c'est précisément parce qu'elle sait reconnaître l'incomplétude de son savoir que la traduction s'ouvre ainsi à de nouvelles pistes de recherche.

\section{NOTES}

1. Dans «La Genèse» de la TOB, on lit: «Lorsque Dieu commença la création du ciel et de la terre, la terre était déserte et vide, et la ténèbre à la surface de l'abîme; le souffle de Dieu planait à la surface des eaux.» Ce souffle est l'Esprit de Dieu ou un vent violent.

2. «Là où commence le chaos s'arrête la science classique.» (Gleick 1991: 18)

3. C'est alors tout l'univers de la mécanique quantique qui s'ouvre et que parcourront Albert Einstein, Niels Bohr et Arnold Summerfeld.

4. On sait maintenant, du moins, il n'est pas trop tard pour l'apprendre, que chaque flocon est unique: étonnamment, il n'y en a pas deux pareils. «Lorsqu'un flocon de neige en formation tombe vers la Terre - flottant dans le vent durant environ une heure -, [...] la nature de l'air turbulent est telle que deux flocons quelconques suivront des trajectoires très différentes. La forme finale d'un flocon est ainsi l'histoire de toutes les variations de conditions atmosphériques qu'il a subies, et leur combinaison peut être pratiquement infinie.» (Gleick 1991: 389-392)

5. La différance chez Derrida signifie que le sens se construit toujours par le rapport qu'entretiennent les signes entre eux. Le rapport ou le jeu interminable entre les traces graphiques confirme que le sens est toujours advenant, donc différé et non présent à lui-même, ce qu'indique le «a » dans le mot différance.

6. Encore faut-il préciser que l'ordre ne se pose pas dans ce contexte-ci comme un concept à valeur universelle dont on pourrait extraire un théorème; il est davantage ponctuel et local. L'idée d'ordre doit donc être comprise comme ce qui permet de discerner des cycles ou des configurations, lesquels sont insolites et changeants, tels les attracteurs étranges et les formes fractales.

7. L'attracteur est la représentation graphique de la trajectoire d'un corps en mouvement qui a ceci de particulier qu'elle ne se recoupe jamais, c'est-à-dire qu'à aucune de ses phases le mouvement ne se répète-t-il identiquement, tout en convergeant en certains points. On dit étrange le fait que les systèmes non linéaires sont à la fois imprévisibles et configurés, tel que le montre l'attracteur étrange en forme d'ailes de papillon qu'Edward Lorenz a produit en 1963. La figure A en annexe illustre comment les spirales ne se rejoignent pas et se divisent en couches disjointes, sans toutefois s'éparpiller en dehors de la figure en forme de papillon.

8. Ainsi, l'imprédictibilité du système météorologique s'explique par l'interaction d'une multitude de facteurs que sont les gaz dans l'atmosphère (rayonnement, conduction, convection, compression, détente, condensation, évaporation), l'échange énergétique, les phénomènes astronomiques, les courants marins, le relief terrestre, la force de Coriolis, les agents polluants, etc.

9. Si l'être humain est souvent porté à universaliser les résultats de ses découvertes, c'est qu'il ne vit pas assez longtemps pour les voir infirmer. Ainsi, s'il nous était donné de vivre 3000 ans, nous constaterions l'effet irrémédiable mais quotidiennement imperceptible de l'un des plus grands systèmes chaotiques qu'est le champ magnétique de la Terre, dont les pôles bougent sans cesse jusqu'à leur renversement total. Les cartes de navigation maritime font état des variantes annuelles longitudinales et latitudinales des pôles magnétiques. De plus, l'alignement magnétique des composantes métalliques de très anciennes pierres volcaniques a prouvé que le pôle sud a déjà été au Nord.

10. C'est encore à Poincaré que revient cette idée.

11. Gleick définit la turbulence ainsi: «Un sacré désordre à toutes les échelles, de petits tourbillons à l'intérieur de grands tourbillons. Elle est instable. Elle est fortement dissipative: elle pompe l'énergie et crée du frottement. Elle est un mouvement devenu aléatoire.» (1991: 160-161)

12. Le site Internet <www.abarim-publications.com/artctmbs.html $>$ présente une animation de l'ensemble de Mandelbrot illustrant le phénomène d'imbrication des formes. Voir la figure B en annexe. 
13. Alors que la géométrie euclidienne permet de concevoir des objets réels selon les dimensions 0 (la droite), 1 (le point), 2 (le plan) et 3 (l'espace), la géométrie fractale donne à voir ce qui se produit lorsque ces dimensions sont fractionnées.

14. L'appareil circulatoire du corps humain, de l'aorte jusqu'aux capillaires, est un exemple extraordinaire d'une surface énorme dans un espace limité. Invraisemblablement, « [1] es poumons d'un être humain moyen renferment une surface supérieure à celle d'un court de tennis» (Gleick 1991: 144).

15. Voir la note 16.

16. «L'estimation de la longueur de la côte anglaise par un observateur à bord d'un satellite sera inférieure à celle d'un observateur parcourant ses criques et ses plages, qui, à son tour, trouvera un résultat inférieur à celui d'un escargot escaladant tous les galets.» (Gleick 1991: 129)

17. Le site Internet $<$ http ://fractales.free.fr/javas/principe/pr.htm $>$ offre une simulation du principe du calcul fractal à partir du flocon de Koch. Ce flocon s'obtient assez facilement: «Imaginez un triangle équilatéral de 30 centimètres de côté. [...] Prenez le tiers central de chacun des côtés, et accolez-lui un nouveau triangle, de forme identique mais de dimensions trois fois plus petites. On obtient une étoile de David. [...] Prenez maintenant chacun des douze côtés et répétez l'opération, en fixant un triangle plus petit sur leur tiers central, et ainsi de suite jusqu'à l'infini.» (Gleick 1991: 132-133) Voir la figure $\mathrm{C}$ en annexe.

18. Voir la figure $\mathrm{D}$ en annexe.

19. Cette proposition rejoint la pensée d'Umberto Eco, selon laquelle « [1]a signification est d'autant plus claire et sans équivoque qu'on s'attache davantage à des règles de probabilité, à des principes d'organisation préétablis et repris dans la répétition d'éléments prévisibles. Inversement, plus la structure devient improbable, ambiguë, imprévisible, désordonnée, plus l'information augmente $[\ldots]$ » (1965).

20. On a récemment annoncé la masse du trou noir situé au centre de notre galaxie: il pèserait 3 millions de masses solaires et se trouverait à 26000 années-lumières, donc actif à l'époque Cro magnon.

21. Celle-ci rejoint le phénomène de l'outre-langue, dont on trouve une définition éloquente dans les Métissages (Nouss et Laplantine 2001: 470-472).

22. «Looked at from any angle, the impact of the processes and concepts of chaos science on the creative arts and the global village of modern popular culture is by now so widespread that it should not be ignored by critical or cultural theorists, students and teachers of literature.» (Hawkins 1995: 10)

23. Dans son introduction, N. Katherine Hayles esquisse le parcours fascinant du chaos à travers l'histoire occidentale (1990). Soulignons également l'interrelation qui unit la science et la littérature dans la science-fiction.

24. Il faut préciser «aujourd'hui», car les sciences humaines sont passées par une période d'abstraction théorique au cours de leurs années structuralistes.

25. Il s'agit du système de signifiance chez Meschonnic, selon lequel le sujet qui lit et traduit organise et désorganise les marques qui pour lui font sens dans un texte.

26. Entre autres penseurs du désordre, Paul-Antoine Miquel réfléchit sur cette troisième voie, tablant sur l'importance de faire du réel un ordre à quoi le désordre participe. Ainsi il écrit: «Or dans ce monde où les objets obéissent à une structure invariante qui explique leurs mouvement ou leurs changements, je jette à présent de l'imprévisible, de la stochasticité, du désordre. Cela veut dire que ce qui à un niveau est en ordre est en même temps, à un autre niveau, en désordre. Comment concevoir une telle chose sans accorder une positivité au désordre? Si je l'admets, il est bien autre chose qu'un négatif qu'il faut opposer au positif.» (Miquel 2000: 10-11)

27. "Chaos theory is a wide-ranging interdisciplinary research front that includes work in such fields as nonlinear dynamics, irreversible thermodynamics, meteorology, and epidemiology.» (Hayles 1990: 9)

\section{RÉFÉRENCES}

Berman, A. (1989): «La traduction et ses discours», dans Meta 34-4, p. 672-679.

Derrida, J. (1972): Positions, Paris, Éditions de Minuit.

Eco, U. (1965): L'œuvre ouverte, Éditions du Seuil.

Gleick, J. (1991): La théorie du chaos (traduit par Christian Jeanmougin), Paris, Flammarion.

Hawkins, H. (1995): Strange Attractors, New York, Prentice Hall/Harvester Wheatsheaf.

Hayles, N. K. (1990): Chaos Bound, Ithaca, Cornell University Press.

Kunn, T. (1983): La structure des révolutions scientifiques, Paris, Flammarion. 
130 MetA, LI, 1, 2006

Meschonnic, H. (1982): Critique du rythme. Anthropologie historique du langage, Lagrasse, Éditions Verdier.

Miquel, P.-A. (2000): Comment penser le désordre?, Paris, Fayard.

Nouss, A. et F. Laplantine (2001): Métissages, Paris, Pauvert. 